\title{
More than two-thirds of dietary iodine in children in northern Ghana is obtained from bouillon cubes containing iodized salt
}

\author{
Abdul-Razak Abizari ${ }^{1}$, Susanne Dold ${ }^{2}$, Roland Kupka ${ }^{3}$ and Michael B Zimmermann ${ }^{2}$ * \\ ${ }^{1}$ Department of Community Nutrition, School of Allied Health Sciences, University for Development Studies, Tamale, \\ Ghana: ${ }^{2}$ Human Nutrition Laboratory, Institute of Food Science and Nutrition, Swiss Federal Institute of Technology, \\ LFVD20, Schmelzbergstrasse 7, CH-8092 Zurich, Switzerland: ${ }^{3}$ UNICEF, Nutrition Section, New York, NY, USA
}

Submitted 25 January 2016: Final revision received 30 August 2016: Accepted 27 September 2016: First published online 1 December 2016

\begin{abstract}
Objective: Bouillon cubes are widely consumed by poor households in sub-Saharan Africa. Because their main ingredient is salt, bouillon cubes could be a good source of iodine if iodized salt is used in their production and if their consumption by target groups is high. Our objective was to measure the iodine content of bouillon cubes, estimate their daily intake in school-aged children and evaluate their potential contribution to iodine intakes.

Design: In a cross-sectional study, we measured urinary iodine concentrations (UIC) and estimated total daily iodine intakes. We administered a questionnaire on usage of bouillon cubes. We measured the iodine content of bouillon cubes, household salt, drinking-water and milk products.

Setting: Primary schools in northern Ghana.

Subjects: Schoolchildren aged 6-13 years.

Results: Among school-aged children ( $n$ 250), median (interquartile range) UIC and estimated iodine intake were $242(163-365) \mu \mathrm{g} / \mathrm{l}$ and $129(85-221) \mu \mathrm{g} / \mathrm{d}$, indicating adequate iodine status. Median household salt iodine concentration ( $n$ 100) was only $2 \cdot 0(0 \cdot 83-7.4) \mu \mathrm{g} / \mathrm{g} ; 72 \%$ of samples contained $<5 \mu \mathrm{g}$ iodine/g. Iodine concentrations in drinking-water and milk-based drinks were negligible. Median iodine content of bouillon cubes was $31.8(26.8-43.7) \mu \mathrm{g} / \mathrm{g}$, with large differences between brands. Estimated median per capita consumption of bouillon cubes was $2 \cdot 4(1 \cdot 5-3 \cdot 3) \mathrm{g} / \mathrm{d}$ and median iodine intake from bouillon cubes was 88 (51-110) $\mu \mathrm{g} / \mathrm{d}$.

Conclusions: Despite low household coverage with iodized salt, iodine nutrition in school-aged children is adequate and an estimated two-thirds of their dietary iodine is obtained from bouillon cubes.
\end{abstract}

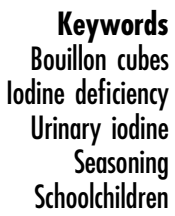

Salt iodization is a highly effective fortification strategy in most countries, including many in Africa ${ }^{(1)}$. Ensuring high household coverage with adequately iodized salt is a key to success of these programmes ${ }^{(1)}$. However, in some countries, in areas where there are multiple scattered small-scale salt producers, it is difficult to establish the infrastructure for salt iodization. This is the case in several countries in West Africa, including Senegal and Ghana ${ }^{(2)}$. In Ghana, since 2011, household coverage of adequately iodized salt ( $\geq 15 \mathrm{ppm}$ ) has been $35-48 \%$, while the proportion of household salt with any concentration of iodine has been $66-68 \%^{(3,4)}$ (J Knowles, unpublished results).

In many industrialized countries, processed foods account for more than $80 \%$ of dietary salt intake ${ }^{(5)}$. Salt used in those foods needs to be iodized if salt iodization programmes are to provide sufficient iodine to target populations ${ }^{(5)}$. Similarly, in low- and middle-income countries with growing processed food consumption ${ }^{(6)}$, iodizing household salt alone may not be sufficient to ensure adequate population iodine status. In West Africa, processed foods provide increasing amounts of dietary salt, especially in urban areas ${ }^{(7)}$. One important processed food product is bouillon cubes. Bouillon cubes are typically composed of 50-70\% salt, along with dehydrated stock, solid vegetable fat, flavour enhancers and spices ${ }^{(8)}$; they are marketed in a variety of flavours such as vegetable, seafood, chicken and beef. Bouillon cubes can be 'indirectly' fortified with iodine through the use of iodized salt in their production ${ }^{(7)}$. Consumption of bouillon cubes containing iodized salt may be increasing in countries in Central and Western Africa, Asia and, more recently, in the Caribbean and Latin America ${ }^{(7,8)}$. 
In Africa, bouillon cubes are widely available in both rural and urban markets and at a low cost that makes them accessible to poorer subgroups of the population ${ }^{(7)}$. In Senegal, the estimated per capita daily consumption of bouillon cubes is $4.3 \mathrm{~g}$ in rural areas ${ }^{(7)}$. Therefore, the use of iodized salt in bouillon could be an effective vehicle and strategy to complement household iodized salt. A potential advantage of the use of iodized salt in bouillon cubes within West Africa is that the bouillon industry is consolidated, in contrast to small-scale salt production, making regulation and monitoring more feasible.

Current legislation on salt iodization in Ghana stipulates that all food-grade salt sold in the country must be fortified with iodine at a manufacturer level of $50 \mathrm{ppm}^{(2)}$. Many bouillon cube manufacturers supplying the Ghanaian market, located both within and outside Ghana, reported complying with this legislation (A-R Abizari, unpublished results). Despite low household coverage with iodized salt in northern Ghana, the recent national survey of iodine nutrition based on median urinary iodine concentration (UIC) found adequate iodine intakes in school-aged children, with the median UIC greater than $150 \mu \mathrm{g} / \mathrm{l}$ (J Knowles, unpublished results). We therefore conducted a cross-sectional survey to assess iodine from different dietary sources and hypothesized that iodized salt in bouillon cubes is the major source of iodine in rural diets of school-aged children in northern Ghana.

\section{Methods}

\section{Study site and participants}

The current cross-sectional survey was conducted in Bole and Sawla communities, located in two neighbouring districts of the western corridor of Northern Region, Ghana. The region is within the Savannah agro-ecological zone and largely rural. Most available food is produced through subsistence farming. In each community, one public primary school was selected to participate. Primary schools were selected in collaboration with the district education directors in order to include children representative of the usual sociodemographic characteristics of the communities. We selected schools that were not participating in the national school feeding programme, to maximize the chances that most of the children's meals were consumed at home. The primary schooling rate estimated from the 2010 population and housing census was $51 \%$ in Bole district $^{(9)}$ and $59 \%$ in Sawla-Tuna-Kalba district ${ }^{(10)}$.

Pupils in lower primary (classes 1-4, age 6-13 years) were selected to participate in the survey. The $a$ priori decision was for 250 pupils to be included in the survey, in order to provide a population-level estimate of iodine status in this age group. In each school, the class registers were pooled to form one sampling frame. Simple random sampling was used to select 125 pupils from each school. The study was conducted in April 2014 with a follow-up in April 2015.

\section{Data collection}

A short questionnaire (pertaining to age, sex, class, date of birth, weight and height) was completed for each selected pupil in school. Each pupil collected a midstream urine sample into a $20 \mathrm{ml}$ sterile screw-top urine container and the samples were transported to the Public Health Laboratory of the Tamale Teaching Hospital, Ghana, where they were aliquoted into $2 \mathrm{ml}$ tubes and stored at $-20^{\circ} \mathrm{C}$. Of the pupils who returned a spot urine sample, 100 were randomly selected and given containers to bring salt samples from their households the next day. The salt samples were stored in opaque polyethylene bags until analysis.

An additional 100 pupils were randomly selected and followed home to complete a questionnaire on household utilization of bouillon cubes. Mothers/caregivers of the pupils, who had primary responsibility for cooking meals, were asked whether they add bouillon cubes to the foods the pupils eat at home. A $24 \mathrm{~h}$ recall of bouillon utilization was conducted. Mothers/caregivers were asked to recall whether bouillon cubes were used for each cooking moment within the $24 \mathrm{~h}$ preceding the interview. The number of bouillon cubes used per cooking moment and the frequency of cooking moments per day were recalled. To have an idea of how regularly the households used bouillon cubes, respondents were asked to indicate the usual frequency of bouillon utilization in a week. Respondents also provided information about the favourite brands of bouillon cubes used by their households.

Samples ( $n$ 34) of the different brands of bouillon cubes available in the communities were bought from different retailers at the respective markets. We sampled the three major brands produced in Ghana (Brands A, B and C; $n$ 27) and seven minor brands ( $n$ 9). Household water from different sources was collected (all, $n$ 17): borehole ( $n$ 5); pipe borne ( $n 7)$; well ( $n$ 3) and dam ( $n 2)$. Milk products $(n 7)$ sold to children (locally made on a small scale) were collected. Urine, salt, food and water samples were sent to the Human Nutrition Laboratory of the ETH Zurich, Switzerland for analysis.

\section{Antbropometry}

Weight and height of children were measured according to standard procedures ${ }^{(11)}$. Height was measured to the nearest $0 \cdot 1 \mathrm{~cm}$ with a microtoise (Bodymeter 208; Seca $\mathrm{GmbH}$, Germany). Weight was measured with a calibrated electronic scale (UNIscale; Seca $\mathrm{GmbH}$ ) to the nearest $0 \cdot 1 \mathrm{~kg}$. Both weight and height were measured twice for each child and the average of the two measurements was taken. Age was calculated using verifiable records (birth certificate, health record, class register) or, in a few cases, estimated based on another child's record or event on a traditional calendar.

\section{Laboratory analyses}

UIC was measured at ETH Zurich using the Pino modification of the Sandell-Kolthoff reaction ${ }^{(12)}$. The iodine laboratory at 
the ETH Zurich participates successfully in the EQUIP programme of the US Centers for Disease Control and Prevention, Atlanta, GA, USA. Iodine in household water samples and salt aliquots dissolved in ultrapure water $(18 \mathrm{M} \Omega \mathrm{cm})$ was measured using a modification of the Sandell-Kolthoff reaction; at iodine concentration of $30 \mu \mathrm{g} / \mathrm{g}$ salt, the inter-assay CV in our laboratory is $7 \%$. The iodine concentration of local milk products and bouillon cubes was analysed in duplicate by inductively coupled plasma-mass spectrometry (ICP-MS). Bouillon cubes were dissolved in $400 \mathrm{ml}$ ultrapure water $(18 \mathrm{M} \Omega$ $\mathrm{cm}$ ) using an ultrasonic bath. Sample preparation of the milk product and dissolved bouillon cubes was done using tetramethylammonium hydroxide for iodine extraction at $90{ }^{\circ} \mathrm{C}^{(13)}$. We used a Finnigan NEPTUNE high-resolution double-focusing multicollector ICP-MS (Thermo Scientific, Waltham, MA, USA) and applied isotope dilution analysis with ${ }^{129}$ I for quantification and tellurium for mass bias correction, as recently described ${ }^{(14)}$.

\section{Data management and statistical analysis}

Microsoft ${ }^{\circledR}$ Excel 2010 and IBM SPSS Statistics Version 22.0 were used for the data analysis. Total daily household bouillon cube utilization was estimated by multiplying the quantity of bouillon cube per cooking moment by the number of usual cooking moments in the household. The household daily total consumption of bouillon cubes was divided by the corresponding household size to derive the individual consumption by school-aged children. Estimated iodine intake from bouillon cubes was calculated for each child by multiplying the calculated daily per capita bouillon cube intake by the mean concentration of iodine in the preferred brand of bouillon cube in the child's household. For the estimations of individual-level intakes of bouillon we assumed that: (i) iodine in the bouillon cubes is stable during storage; (ii) there is no loss of iodine during cooking; and (iii) all foods prepared at home and containing bouillon cubes were completely consumed. Estimated total daily iodine intake was calculated from the UIC and body weight of each child using the following US Institute of Medicine ${ }^{(15)}$ formula:

Daily iodine intake $=$ Urinary iodine concentration $(\mu g / 1)$

$$
\times 0.0235 \times \text { body weight }(\mathrm{kg}) \text {. }
$$

Evaluation of Q-Q plots, the Kolmogorov-Smirnov test and the Shapiro-Wilk test were used to test normality of data. In descriptive analyses, normally distributed data were presented as mean and standard deviation, and nonnormally distributed data as median and interquartile range (IQR). UIC, salt iodine concentration (SIC), estimated iodine intake from UIC and the content of iodine in the cubes were not normally distributed. In the case of non-normality, the data were log transformed and rechecked for normal distribution. If data were still not normal after log transformation, non-parametric tests were used for analysis of the non-transformed data. Spearman's correlations were conducted since data were not normally distributed. The level of significance was set at $P<0 \cdot 05$.

\section{Results}

The background characteristics of the children and their caregivers are shown in Table 1 . Among the children ( $n$ 250), mean (SD) age was 9 (1.6) years, 46.8\% were male, and fifty-seven (22.8\%), seventy-three (29.2\%), seventy-eight (31.2\%) and forty-two (16.8\%) were from primary school classes 1, 2, 3 and 4, respectively. Mean (SD) household size was 8 (4), 74\% were Muslim, and the literacy rate among the caregivers was $28 \%$.

The SIC in household samples and the children's UIC values are shown in Table 2 . The median SIC was only approximately $2 \mu \mathrm{g} / \mathrm{g}$ : $72 \%$ of samples had SIC $<5 \mu \mathrm{g} / \mathrm{g}, 6 \%$ had SIC between 5 and $15 \mu \mathrm{g} / \mathrm{g}, 4 \%$ between 15 and $40 \mu \mathrm{g} / \mathrm{g}$,

Table 1 Characteristics of northern Ghanaian schoolchildren and their caregivers, April 2014

\begin{tabular}{lrrr}
\hline Characteristic & $\%$ & Mean & SD \\
\hline Children $(n$ 250) & & & \\
Male (\%) & $46 \cdot 8$ & & \\
Age (years) & & 27.4 & 1.6 \\
Weight (kg) & & $130 \cdot 6$ & $9 \cdot 1$ \\
$\quad$ Height (cm) & & & \\
Mothers/caregivers ( $n$ 100) & & & \\
Education (\%) & 28 & & \\
$\quad$ Formal education & 72 & & \\
$\quad$ No formal education & & & \\
Religion (\%) & 74 & & \\
$\quad$ Muslim & 24 & & \\
$\quad$ Christian & 2 & & \\
$\quad$ Other & & & \\
Occupation (\%) & 15 & & \\
$\quad$ Farmer & 65 & & \\
$\quad$ Trader & 4 & & \\
$\quad$ Civil servant & 16 & & \\
$\quad$ Other & & & \\
Household size & & & \\
\hline
\end{tabular}

Table 2 Household salt iodine concentrations and iodine status of northern Ghanaian schoolchildren, April 2014

\begin{tabular}{|c|c|c|c|c|}
\hline & Median & IQR & $n$ & $\%$ \\
\hline \multicolumn{5}{|l|}{$\operatorname{SIC}(n 100)$} \\
\hline SIC (mg/kg) & 1.9 & $0.83-7.4$ & & \\
\hline \multicolumn{5}{|l|}{ SIC distribution (\%) } \\
\hline$<5 \mathrm{mg} / \mathrm{kg}$ & & & & 72 \\
\hline $5-<15 \mathrm{mg} / \mathrm{kg}$ & & & & 6 \\
\hline $15-40 \mathrm{mg} / \mathrm{kg}$ & & & & 4 \\
\hline$>40 \mathrm{mg} / \mathrm{kg}$ & & & & 18 \\
\hline \multicolumn{5}{|l|}{ UIC (n 246) } \\
\hline UIC $(\mu \mathrm{g} / \mathrm{l})$ & 241.9 & $162 \cdot 5-364 \cdot 5$ & & \\
\hline \multicolumn{5}{|l|}{ UIC distribution } \\
\hline$<50 \mu \mathrm{g} / \mathrm{l}$ & & & 7 & $2 \cdot 8$ \\
\hline $50-99 \mu \mathrm{g} / \mathrm{l}$ & & & 18 & $7 \cdot 3$ \\
\hline $100-299 \mu \mathrm{g} / \mathrm{l}$ & & & 134 & 54.5 \\
\hline$\geq 300 \mu \mathrm{g} / \mathrm{l}$ & & & 87 & $35 \cdot 4$ \\
\hline
\end{tabular}

IQR, interquartile range; SIC, salt iodine concentration; UIC, urinary iodine concentration. 
Table 3 lodine concentration of bouillon cubes ( $n$ 34) sold in northern Ghana, April 2014

\begin{tabular}{|c|c|c|c|}
\hline & \multirow[b]{2}{*}{$n$} & \multicolumn{2}{|c|}{ lodine concentration $(\mu \mathrm{g} / \mathrm{g})$} \\
\hline & & Median & IQR \\
\hline All bouillon tablets & 34 & $\begin{array}{l}27 \cdot 2 \\
31 \cdot 8^{*}\end{array}$ & $\begin{array}{l}17 \cdot 5-31 \cdot 4 \\
26 \cdot 8-43 \cdot 7\end{array}$ \\
\hline \multicolumn{4}{|l|}{ Brands of bouillon } \\
\hline Brand A & 9 & $32 \cdot 0$ & $27 \cdot 2-47 \cdot 5$ \\
\hline Brand B & 8 & $30 \cdot 6$ & $25 \cdot 6-31 \cdot 5$ \\
\hline Brand C & 8 & $27 \cdot 6$ & $27 \cdot 0-29 \cdot 2$ \\
\hline Others & 9 & 9.6 & $0.2-10 \cdot 8$ \\
\hline
\end{tabular}

IQR, interquartile range.

*Weighted by frequency of consumption of brands.

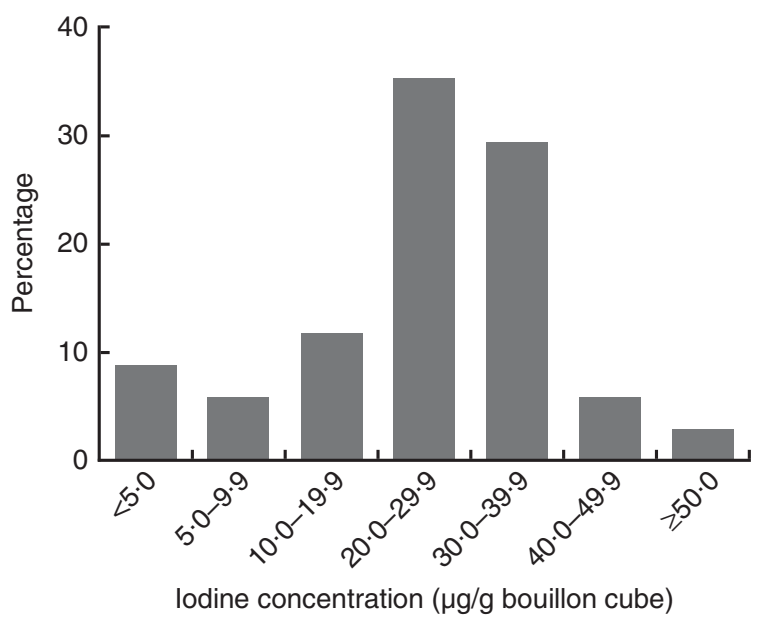

Fig. 1 Distribution of iodine concentration in bouillon cubes sold in northern Ghana, April 2014

and $18 \%$ had SIC $>40 \mu \mathrm{g} / \mathrm{g}$. The median (IQR) UIC of the schoolchildren was 242 (163-365) $\mu \mathrm{g} / \mathrm{l}$ (Table 2).

The iodine concentrations in household water and local milk products were low. The median (IQR) iodine concentration in household water $(n 17)$ was $0 \cdot 0(0 \cdot 0-3 \cdot 0) \mu \mathrm{g} / \mathrm{l}$; one water sample from a borehole contained $35 \mu \mathrm{g} / 1$ and one well water sample contained $27 \mu \mathrm{g} / \mathrm{l}$; all other samples contained $<5 \mu \mathrm{g} / \mathrm{l}$. The median (IQR) iodine concentration in milk products $(n$ ) ) was 9.0 (8.3-9.0) $\mathrm{ng} / \mathrm{g}$.

Except for Brand A, which was packed as $12 \mathrm{~g} / \mathrm{cube}$, all other brands of bouillon cube were packed as $10 \mathrm{~g} / \mathrm{cube}$. Among the study households, there was a clear preference for Brand A cubes (used by $75 \%$ of households), while $22 \%$ of households used Brand B cubes and only $3 \%$ preferred other brands. The overall median (IQR) concentration of iodine in bouillon cubes ( $n$ 34), weighted by frequency of brand use, was $31.8(26 \cdot 8-43.7) \mu \mathrm{g} / \mathrm{g}$. By brand, the median (IQR) concentration of iodine in the three most popular brands of bouillon cubes was: Brand A $(n$ 9), 32.0 $(27 \cdot 2-47.5) \mu \mathrm{g} / \mathrm{g} ;$ Brand B $(n$ 8), 30.6 $(25 \cdot 6-31 \cdot 5) \mu \mathrm{g} / \mathrm{g}$; and Brand C ( $n$ 8), $27 \cdot 6$ (27.0-29.2) $\mu \mathrm{g} / \mathrm{g}$ (Table 3). The distribution of iodine concentrations $(\mu \mathrm{g} / \mathrm{g})$ among all samples of bouillon cubes is shown in Fig. 1.
Table 4 Household bouillon cube use and estimated contribution of bouillon cubes to daily iodine intake in northern Ghana, April 2014 (n 100)

\begin{tabular}{lrc}
\hline & Median & IQR \\
\hline No. of bouillon cubes per cooking moment & 1.0 & $1.0-1.0$ \\
No. of bouillon cubes used per day & 2.0 & $1.0-2.0$ \\
Quantity of bouillon cube used per day $(\mathrm{g})$ & 20.0 & $12.0-24.0$ \\
Per capita bouillon cube intake $(\mathrm{g} / \mathrm{d})$ & 2.4 & $1.5-3.3$ \\
Estimated per capita iodine intake from & 88.3 & $50.9-110.4$ \\
$\quad$ bouillon cubes $(\mu \mathrm{g} / \mathrm{d})$ & & \\
Estimated total per capita iodine intake & 129.3 & $85.1-220.9$ \\
$\quad$ based on UIC $(\mu \mathrm{g} / \mathrm{d})$ & & \\
\hline
\end{tabular}

IQR, interquartile range; UIC, urinary iodine concentration.

Among the households surveyed ( $n$ 100), the median (IQR) quantity of bouillon cubes used daily was $20 \cdot 0$ $(12 \cdot 0-24 \cdot 0) \mathrm{g}$ per household and the per capita daily bouillon cube intake was estimated at $2 \cdot 4(1 \cdot 5-3 \cdot 3) \mathrm{g}$. Weighted by frequency of use of the different brands among the households (above), we calculated the estimated median (IQR) per capita iodine intake from bouillon cubes as $88.3(50 \cdot 9-10 \cdot 4) \mu \mathrm{g} / \mathrm{d}$. Median (IQR) daily iodine intake projected from the children's UIC and body weight was $129 \cdot 3(85 \cdot 1-220 \cdot 9) \mu \mathrm{g}$ (Table 4). There was no significant correlation between SIC and UIC, after controlling for iodine intake from bouillon cubes, but there was a significant positive correlation between bouillon cube iodine content and UIC $(r=0 \cdot 36, P=0 \cdot 031)$.

\section{Discussion}

Our findings suggest that in this region of northern Ghana the major source of iodine in school-aged children's diets is iodized salt used in the manufacture of bouillon cubes. Despite very low coverage of households with iodized salt, iodine intakes were adequate, as the median iodine intake, derived from the UIC of the children, was $129 \mu \mathrm{g} / \mathrm{d}$, just above the recommended daily iodine intake of $120 \mu \mathrm{g} / \mathrm{d}$ for this age $\operatorname{group}^{(1,15)}$. We estimate that iodine from bouillon cubes was contributing over two-thirds of dietary iodine in the children's diets. In this inland area far from the Atlantic coast, intakes of iodine-rich seafood and ocean fish are negligible, and the groundwater contains very little iodine. Therefore, iodized table salt would be expected be the primary source of dietary iodine. However, our data suggest only $22 \%$ of households were using adequately iodized salt (as defined by SIC $\geq 15 \mathrm{mg} / \mathrm{kg}$ ) ${ }^{(1)}$. Milk products can be a rich source of iodine in countries where diets are rich in dairy products, such as the USA, Switzerland and Australia ${ }^{(5)}$. However, dairy products are consumed only rarely by rural children in northern Ghana, and the concentrations of iodine in milk drinks for sale in the study area were negligible.

Because their main ingredient is salt, bouillon cubes (and other seasoning powders) could be a good fortification 
vehicle for iodine if they contained iodized salt and their consumption by target groups is sufficiently high. Hess et $a l .{ }^{(16)}$ summarized the findings on consumption of bouillon cubes from the Fortification Rapid Assessment Tool (FRAT) surveys conducted in sub-Saharan Africa. Five of the FRAT surveys included bouillon cubes as a food vehicle of interest ${ }^{(16)}$. In Mali, approximately $98 \%$ of all women reportedly consumed bouillon cubes in the previous $24 \mathrm{~h}^{(16)}$. In Burkina Faso in 1999 ( $\left.n 840\right), 79 \%$ of women consumed bouillon cubes in the past week, the mean number of days bouillon cubes were consumed per week was 5.4, and the median amount of bouillon cube consumed on the previous day among consumers was $2 \cdot 6 \mathrm{~g}^{(16)}$. For Niger in 2001 ( $n$ 840), the respective numbers were $88 \%, 5.6$ and $1.7 \mathrm{~g} / \mathrm{d}^{(16)}$. For Senegal in $2006(n 827)$, the respective numbers were $99 \%, 6.8$ and $3.5 \mathrm{~g} / \mathrm{d}^{(16)}$. Finally, for Cameroon ( $n$ 912), the respective numbers were $96 \%, 13.8$ times per week and $1.9 \mathrm{~g} / \mathrm{d}^{(17)}$. In our study population, the estimated median (IQR) per capita bouillon cube intake was $2 \cdot 4(1.5-3.3) \mathrm{g} / \mathrm{d}$, comparable to these previous studies in West Africa.

In our study area, over $70 \%$ of households were using the Brand A of bouillon cube, while about $20 \%$ were using the Brand B. These two popular brands had a median (IQR) iodine concentration of $32.0 \quad(27.2-47.5)$ and $30.6(25.6-31.5) \mu \mathrm{g} / \mathrm{g}$, respectively, and bouillon cubes are usually $\approx 50 \%$ salt $^{(7,8)}$. This suggests that adequately iodized salt, likely close to the Ghanaian standard of $50 \mu \mathrm{g}$ iodine/g, was being used in their manufacture. A recent study in Senegal estimated that, based on the measured iodine content of bouillon cubes and average daily bouillon cube consumption of $4.3 \mathrm{~g}$ in rural areas, bouillon cubes could deliver between 3 and $57 \%$ of the recommended adequate daily iodine intake in adults depending on the brand of bouillon cube consumed ${ }^{(7)}$. That study suggested that bouillon cubes containing salt iodized close to the level stipulated in Senegalese legislation $(30 \mu \mathrm{g} / \mathrm{g})^{(18)}$ could potentially cover $46-57 \%$ of an adult's recommended daily iodine intake of $150 \mu \mathrm{g} / \mathrm{d}^{(7)}$. It should be mentioned, however, that only two out of thirteen brands of bouillon cubes the authors worked with appeared to have been made from adequately iodized salt. Nevertheless, this indicates that bouillon cubes, if they contain well-iodized salt, can be a substantial contributor to iodine adequacy among vulnerable population groups in West Africa such as children and women of reproductive age.

This substantial contribution of 'hidden' iodine in bouillon cubes and other seasoning powders to dietary iodine intakes may explain the discrepancy in many low- to middle-income countries between data suggesting, on the one hand, low coverage of households with adequately iodized salt, but on the other, adequate iodine intake based on the median UIC. Iodine sufficiency in populations as indicated by a median UIC of $\geq 100 \mu \mathrm{g} / \mathrm{l}$ in school-aged children ${ }^{(1)}$ has been reported from many countries with poor household iodized salt coverage. For example, in Senegal, household coverage of adequately iodized salt was $\approx 56 \%$ but median UIC among schoolaged children was in the adequate range $(104 \mu \mathrm{g} / \mathrm{l})^{(18)}$. In the Philippines, household coverage with adequately iodized salt was only $25 \%$ but median UIC in school-aged children was adequate, at $132 \mu \mathrm{g} / \mathrm{l}^{(19)}$. Recognizing this issue, the WHO has stated that countries that focus on iodization of table salt alone may not achieve optimal iodine nutrition and it is necessary to include iodized salt in processed foods ${ }^{(20)}$. This statement by WHO is a reminder to stay true to universal salt iodization, which in principle means that all edible salt, including that used in processed foods, be adequately iodized.

Stability of iodine in poor-quality, damp salt is a concern, and may be partly responsible for the large proportion of inadequately iodized salt at the household level ${ }^{(21)}$ in counties where there is a high percentage of raw, poor-quality salt. Due to the relatively long shelf-life of bouillon cubes and other seasoning powders, similar concerns about iodine losses may also apply to these products albeit the consistency of bouillon cubes is different from moist salt. There is little information on iodine losses in cubes; however, recent studies in Senegal ${ }^{(7)}$ have shown that iodine concentration in bouillon cubes is minimally affected by prolonged storage (average loss of iodine in bouillon cubes was $13.6 \%$ over 6 months) and is virtually unaffected by cooking conditions. This suggests that if producers used adequately iodized salt, bouillon cubes could serve a predictable and stable source of dietary iodine to households. However, data from the bouillon cubes analysed indicated that there are wide differences (up to two- to threefold differences) in iodine concentration within the same brand. Also, there was often disagreement between the analysed values and the concentration stated on the product label. Therefore, there is a need for improved quality control and labelling, and the iodine content in salt used in the preparation of the bouillon cubes will need to be monitored carefully so that its contribution as part of national programmes can be tracked with some certainty.

Fortification of bouillon cubes with iodized salt is a relatively new concept in iodine nutrition programmes. Whether this strategy is successful will depend on the enforcement and willingness of domestic and multinational manufacturers to use iodized salt in their bouillon cubes. Some companies have already voluntarily introduced bouillon cubes with added iodine and iron in Ghana and other parts of Africa. One large manufacturer has released a fortified bouillon product in multiple West African countries (Benin, Togo, Cameroon, Côte d'Ivoire, Nigeria, Senegal, Ghana, Niger and Guinea), which is currently fortified with iodine as well as iron ${ }^{(22)}$. Discussions by the authors with large bouillon cube producers in Ghana revealed that although there are no factory-level iodine fortification protocols, the national regulatory reference of $50 \mathrm{mg} / \mathrm{kg}$ 
guides the procurement of salt they use in bouillon production, and they are willing to comply with legislation regarding iodine fortification. In a recent review, Mejia and Bower ${ }^{(23)}$ recommend that countries with existing voluntary fortification programmes covering bouillon cubes and seasonings revise and convert them into mandatory regulations, to ensure that the products comply with fortification standards and guidelines. Also, government monitoring and enforcement need to be integrated into such mandatory policy guidelines to ensure adherence and compliance $^{(7)}$. Improved labelling would also be valuable: typical packaging refers to the amount of sodium per cube, but does not indicate whether that sodium comes from monosodium glutamate or salt. Therefore, without the product recipe or conducting laboratory analysis, it is impossible to calculate the potential amount of iodine from iodized salt contained per cube solely from the amount of sodium $^{(7)}$. However, the two major brands of bouillon in the current survey listed 'iodated salt' as an ingredient and indicated the amount of iodine per cube. For example, the label of the Brand A bouillon cube package states that $12 \mathrm{~g}$ of the product ( 1 cube) contains $370 \mu \mathrm{g}$ iodine and that of the Brand $\mathrm{B}$ bouillon cube states that $10 \mathrm{~g}$ of the product (1 cube) contains $80 \mu \mathrm{g}$ iodine.

Our study findings have limitations. Estimation of daily intake of bouillon cube per capita was based on the assumption that each meal prepared at the household was completely served to all members of the household and that each schoolchild completely finished his/her portion. Dividing the total quantity of household bouillon cubes used by the household size presumes equal sharing of food but portion sizes usually vary for different individuals in the household, with children receiving smaller portions. This is likely to lead to overestimation of bouillon intake. Nevertheless, our estimate for daily bouillon intake is similar to that derived from a quantitative dietary assessment for schoolchildren within the same region (A-R Abizari, unpublished results). Also, we studied children attending primary schools and only $50-60 \%$ of children in this region are attending school; it is possible that families of children not attending school have different dietary patterns and our findings may not apply to them.

\section{Conclusion}

In conclusion, bouillon cubes are an important source of dietary iodine in northern Ghana and possibly other West African countries. Growing use of bouillon cubes in rural Africa will likely reduce the quantity of table salt used by households during cooking. This could decrease delivery of iodine by household salt even if it is well iodized, and reinforces the need to use adequately iodized salt in the production of bouillon cubes. Future research should determine the potential impact of iodine in bouillon cubes not only on school-aged children in other settings and diets, but also on other target populations of iodine programmes such as pre-school children (who eat less foods from the table) and young women (whose iodine requirements are higher).

\section{Acknowledgements}

Acknowledgements: The authors thank all the children and their mothers/caregivers who participated in the study. They are grateful to the teachers of DA Primary School (Bole and Sawla) for their role in facilitating data collection. They thank Jerry Alagpulinsa, Francis Gakpetor, Mohammed Shaibu Osman, Aboku Osman and Suara Sufyan for helping with data collection and processing of samples, and are thankful to Annica Jucker and Timo Christ for assistance with laboratory analyses. The authors also thank Jacky Knowles for her support and for providing relevant information concerning iodine nutrition in the study area. Financial support: This work was funded by the Global Alliance for Improved Nutrition (GAIN), Geneva, Switzerland; UNICEF, New York, USA; and the Human Nutrition Laboratory, ETH Zurich, Switzerland. The funders had no further role. Conflict of interest: R.K. is a UNICEF staff member. The opinions and statements in this article are those of the author and may not reflect official UNICEF policies. Authorship: A.-R.A. and M.B.Z. designed the research. A.-R.A. conducted and supervised the fieldwork. A.-R.A., S.D. and M.B.Z. analysed the data. A.-R.A. and M.B.Z. wrote the first draft of the paper. All authors made critical comments during the preparation of the manuscript. All authors edited and approved the final version of the manuscript. Ethics of buman subject participation: The parents/caregivers of the pupils gave verbal informed consent. The verbal consent was witnessed and formally recorded. The review boards of the ETH Zurich, Switzerland and the Tamale Teaching Hospital in Ghana gave ethical permission for the study.

\section{References}

1. World Health Organization, UNICEF \& International Council for Control of Iodine Deficiency Disorders (2007) Assessment of Iodine Deficiency Disorders and Monitoring their Elimination: A Guide for Programme Managers, 3rd ed. Geneva: WHO.

2. Nyumuah RO, Hoang T-CC, Amoaful EF et al. (2012) Implementing large-scale food fortification in Ghana: lessons learned. Food Nutr Bull 33, 4 Suppl., 293S-300S.

3. Ghana Statistical Service (2011) Ghana Multiple Indicator Cluster Survey with an Enhanced Malaria Module and Biomarker. Final Report. Accra: GSS.

4. Ghana Statistical Service, Ghana Health Service \& ICF International (2015) Ghana Demographic and Health Survey 2014. Rockville, MD: GSS, GHS and ICF International.

5. Zimmermann MB (2010) Iodine deficiency in industrialized countries. Clin Endocrinol (Oxf) 75, 287-288.

6. Spohrer R, Larson M, Maurin C et al. (2013) The growing importance of staple foods and condiments used as ingredients in the food industry and implications for large-scale 
food fortification programs in Southeast Asia. Food Nutr Bull 34, 2 Suppl., 50S-61S.

7. Spohrer R, Knowles J, Jallier V et al. (2015) Estimation of population iodine intake from iodized salt consumed through bouillon seasoning in Senegal. Ann N Y Acad Sci 1357, 43-52.

8. Mejia EG, Aguilera-Gutiérrez Y, Martin-Cabrejas MA et al. (2015) Industrial processing of condiments and seasonings and its implications for micronutrient fortification. Ann NY Acad Sci 1357, 8-28.

9. Ghana Statistical Service (2014) 2010 Population and Housing Census. District Analytical Report. Bole District. Accra: GSS.

10. Ghana Statistical Service (2014) 2010 Population and Housing Census. District Analytical Report. Sawla-TunaKalba District. Accra: GSS.

11. Cogill B (2003) Anthropometric Indicators Measurement Guide. Washington, DC: Food and Nutrition Technical Assistance Project, Academy for Educational Development.

12. Pino S, Fang S-L \& Braverman LE (1996) Ammonium persulfate: a safe alternative oxidizing reagent for measuring urinary iodine. Clin Chem 42, 239-243.

13. Fecher PA, Goldmann I \& Nagengast A (1998) Determination of iodine in food samples by inductively coupled plasma mass spectrometry after alkaline extraction. J Anal At Spectrom 13, 977-982.

14. Dold S, Baumgartner J, Zeder C et al. (2016) Optimization of a new mass spectrometry method for measurement of breast milk iodine concentrations and an assessment of the effect of analytic method and timing of within-feed sample collection on breast milk iodine concentrations. Thyroid 26, 287-295.

15. Trumbo P, Yates AA, Schlicker S et al. (2001) Dietary reference intakes: vitamin A, vitamin $\mathrm{K}$, arsenic, boron, chromium, copper, iodine, iron, manganese, molybdenum, nickel, silicon, vanadium, and zinc. J Am Diet Assoc 101, 294-301.

16. Hess SY, Brown KH, Sablah M et al. (2013) Results of Fortification Rapid Assessment Tool (FRAT) surveys in sub-Saharan Africa and suggestions for future modifications of the survey instrument. Food Nutr Bull 34, 21-38.

17. Engle-Stone R, Ndjebayi AO, Nankap M et al. (2012) Consumption of potentially fortifiable foods by women and young children varies by ecological zone and socio-economic status in Cameroon. J Nutr 142, 555-565.

18. Ministere de la Sante et de la Prevention Medicale, Université Cheikh Anta Diop \& Micronutrient Initiative (2010) Etude sur les troubles dus a la carence en iode au Senegal. Dakar: Ministere de la Sante et de la Prevention Medicale, UCAD and MI.

19. Food and Nutrition Research Institute (2008) Nutritional Status of Filipino Children and Selected Population Groups: 2005 Update. Manila: FNRI.

20. World Health Organization (2014) Guideline: Fortification of Food-Grade Salt with Iodine for the Prevention and Control of Iodine Deficiency Disorders. Geneva: WHO.

21. Diosady LL, Alberti JO, Venkatesh Mannar MG et al. (1997) Stability of iodine in iodized salt used for correction of iodine-deficiency disorders. Food Nutr Bull 18, 388-396.

22. Nestlé (2015) Nestlé introduces iron-fortified MAGGI bouillons to consumers in Togo and Benin. http://www. nestle-cwa.com/en/nestl-introduces-iron-fortified-maggibouillons-to-consumers-in-togo-and-benin, (accessed June 2015).

23. Mejia LA \& Bower AM (2015) The global regulatory landscape regarding micronutrient fortification of condiments and seasonings. Ann N Y Acad Sci 1357, 1-7. 